\title{
Discussion of Current-Sheet Approximations in Reference to High-Frequency Magnetic Measurements
}

\author{
Bohdan Kostyshyn and Peter H. Haas
}

\begin{abstract}
The problem of violations of thin, uniform current-sheet assumptions in toroidal coils of rectangular cross section is investigated from the viewpoint of high-frequency magnetic measurements. The errors resulting by neglecting leakage flux and by assumptions as to the location of effective current sheets are investigated experimentally as a function of permeability, core dimensions, wire spacing, and wire size. Conclusions are reached as to the adequacy of at least two attempts at calculations of these errors. Finally, conclusions are drawn as to the limitations of measurements on toroidal coils by high-frequency considerations.
\end{abstract}

\section{Definition of Symbols}

$\mu=$ Magnetic permeability of the material (as used in this paper, the initial permeability)

$L_{m}=$ Measured inductance of sample coil containing core.

$L_{S}=$ Calculated value of toroidal coil inductance on basis of uniform thin current-sheet assumptions.

$\mu_{e}=$ Permeability of material as inferred from inductance ratio of measured to calculated inductance

$b, a, h=$ Outside diameter, inside diameter, and height of toroidal core, respectively.

$\mu_{a}=$ Value of $\mu_{e}$ reached as the number of turns becomes very large.

$\Delta \mu=$ Absolute permeability error

$\Delta \mu_{1}: \Delta \mu_{2}=$ Components of $\Delta \mu$, see text.

$x=$ Distance of the effective current sheet from the face of the toroid.

$\Delta L_{m}=$ Correction term to the measured inductance for $\mu_{e}$ to equal $\mu$.

$\Delta L_{S}=$ Correction term to the calculated inductance for $\mu_{e}$ to equal $\mu$.

$D=$ Mean spacing between adjacent wires on toroidal coil.

$\Delta L_{R}=$ Correction term of the type $\Delta L_{S}$ as calculated from theoretical considerations by Rosa.

\section{Introduction}

It has commonly been the practice in the past [1] to determine the initial permeability of a magnetic material at radio frequencies by winding a toroidal sample with $N$ turns of wire, measuring its inductance, $L_{m}$, at low magnetic field strengths, calculating the geometrical inductance, $L_{S}$, of an air toroid of the same dimensions, and taking the ratio of the two, $L_{m} / L_{S}$, to be the permeability. Another method employed [2] has been to substitute for $L_{S}$ the measured value of inductance $\left(L_{m}\right)_{a}$ of an equivalent air toroid wound on a polystyrene core of identical dimensions, with the same wire and number of turns as the magnetic core to be measured.

The assumption in applying the first method is that the given ratio does not depend on the frequency (if the permeability is truly constant), the number of turns wound on the sample, wire size, and the dimensions of the sample core. The assumption in applying the second method is that any corrections that appear for the inductances for the coils containing the magnetic core and the equivalent air core, respectively, are equal and appear in such a manner as to cancel when the ratio is taken. It is the purpose of this paper to test the validity of these assumptions.

\section{Limitations on Test Parameters}

For the test the various parameters were limited such that the following conditions were adhered to:

1. The magnetic field strength was sufficiently low that the permeability $\mu$ measured would be equal to the initial permeability; that is, that value of the permeability obtained as the magnetic induction, $B$, and the magnetic field intensity, $H$, simultaneously approach zero.

2 . The frequency was low enough that capacitance effects from wire proximities could be demonstrated to be negligible, yet it was high enough that the internal inductance of the wire could be neglected.

3 . The magnetic materials were chosen so that the permeability showed no change as a function of frequency until a frequency many times the test frequency was reached.

4. Realistic wire sizes allowing a tight, uniform winding were used. A toroidal winding machine was used (fig. 1) to apply single-layer windings to cores of rectangular cross section.

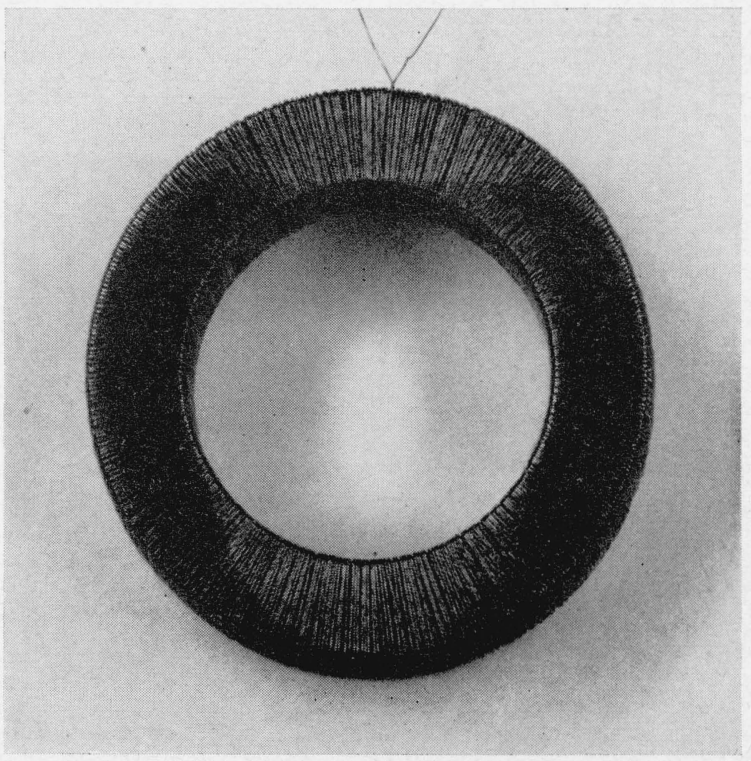

Figure 1. Typical coils used. 


\section{Analysis of Assumptions}

We now define the effective permeability of a toroidal sample core as

$$
\mu_{\bullet}=\frac{L_{m}}{L_{S}}
$$

where $\mu_{e}$ is effective permeability; $L_{m}$ is the measured inductance of sample coil, and $L_{S}$ is the calculated inductance of an air toroid, equivalent to sample coil, assuming a thin, uniform current sheet, where $L_{S}$ is equal to

$$
L_{S}=2 N^{2} h \log _{e} \frac{b}{a} \times 10^{-7} \text { henry, }
$$

where $N$ is the number of turns of wire on sample, $h$ is the height of sample in meters, $b$ is the outer diameter of sample, and $a$ is the inner diameter of sample.

If one now varies the number of turns on a given magnetic core, with given wire size and plots the effective permeability $\mu_{e}$ as a function of $N$, it is observed (see fig. 2) that the assumption that the ratio $L_{m} / L_{S}$ is not dependent on $N$, as assumed earlier, does not hold, but that for small $N$ a large value of $\mu_{e}$ results, and as $N$ is increased, $\mu_{e}$ approaches asymptotically a value $\mu_{a}$ slightly higher than the true permeability $\mu$, as determined by an NBS radiofrequency permeameter [3].

One now recognizes three values for permeability: $\mu$ equals true initial permeability; that value of permeability obtained by using eq (1), provided a thin, uniform current sheet exists adjacent to the toroidal sample.

$\mu_{e}$ equals effective permeability; that value of permeability obtained from eq (1) when the sample core

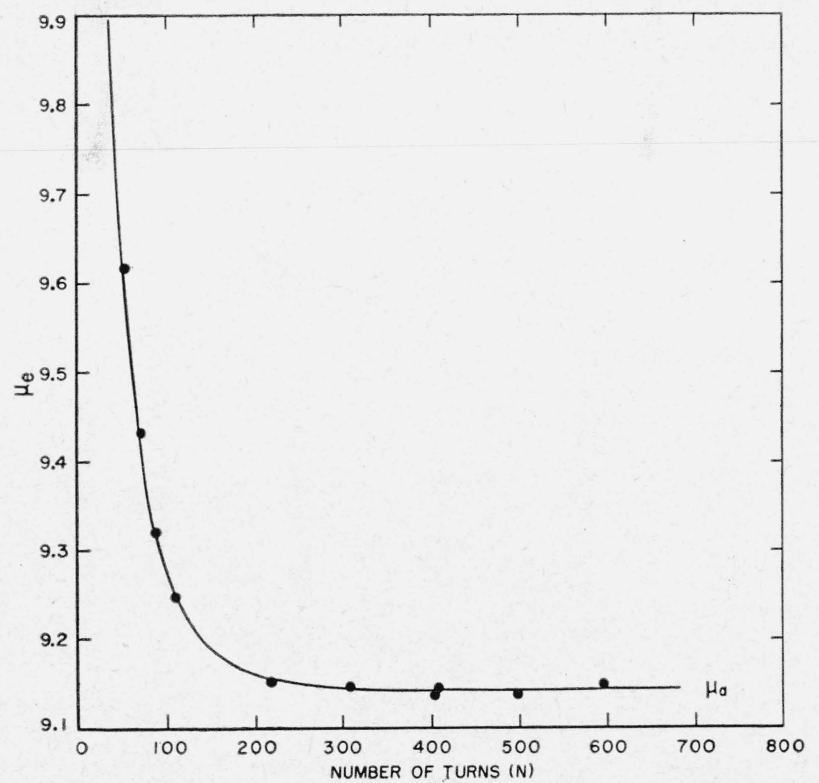

Figure 2. Variation of $\mu_{e}$ with number of turns, $N$.

Wire: Insulated No. 38. Core: Outside diameter, 1.507 in.; inside diameter, 1.004 in.; height, 0.375 in.

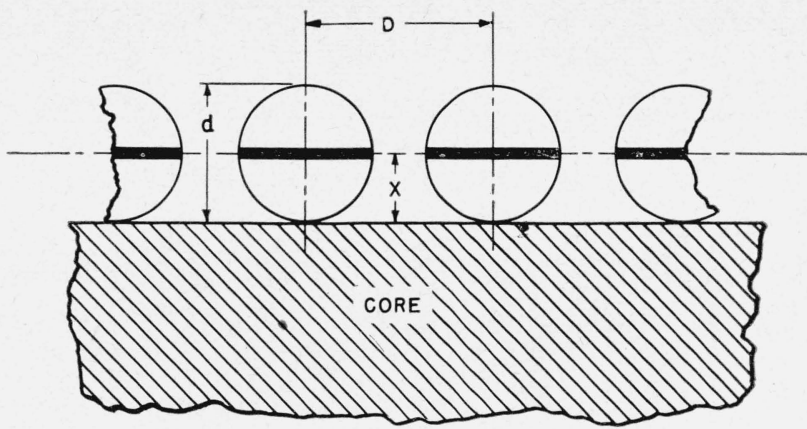

FIGURE 3. Schematic cross section of the surface of a toroidal coil wound with round wire of diameter $d$.

is wound with $N$ turns of actual wire of finite dimensions.

$\mu_{a}$ equals asymptotic permeability; that value of effective permeability reached as the number of turns $N$ becomes very large (fig. 2 ).

We further define

$$
\begin{gathered}
\Delta \mu=\Delta \mu_{1}+\Delta \mu_{2}=\mu_{e}-\mu, \\
\Delta \mu_{1}=\mu_{a}-\mu, \\
\Delta \mu_{2}=\mu_{e}-\mu_{a} .
\end{gathered}
$$

As noted before, the assumption made in applying eq (1) and assuming it to yield the true permeability is that a constant current sheet exists about and immediately adjacent to the toroidal core. This condition requires that there be no external magnetic fields beyond the confines of the current sheet.

For an actual coil wound with wire of finite dimensions and individual turns spaced a finite distance apart (fig. 3) it is seen that a thin current sheet may not exist, but one may visualize a hypothetical current strip in each wire. If one assumes the existence of a definite current distribution, one might calculate a mean or effective current strip, located a finite distance $x$ from the face of the core. It is further noted that as the number of turns is increased the mean wire spacing $D$ is decreased and the current sheet is more nearly approximated. One may predict on this basis that for a few turns the predominant cause for the error $\Delta \mu_{2}$ is the existence of a series of current strips, rather than a current sheet. This then implies the existence of magnetic fields beyond the confines of the core. For a large number of turns one may ascribe the error $\Delta \mu_{1}$ to be caused by the location of an assumed current sheet at some finite distance from the faces of the core, as determined by the current distribution in the wire rather than immediately adjacent to the core.

\section{Discussion of Correction Terms}

It has been demonstrated in the literature $[1,4]$ that corrections might be made to account for these more realistic conditions.

In one case it is assumed that the magnetic flux exists only within the confines of the coil, including 
that space not occupied by the core. The permeability is then calculated from

$$
\mu=\frac{L_{m}-\Delta L_{a}}{L_{S}},
$$

where

$$
\begin{aligned}
\Delta L_{a} & =\frac{4 N^{2} A_{a}}{d_{e}} \times 10^{-7} \text { henry }[1] \\
& =4 N^{2} x \frac{[b-a+4 x+2 h]}{b-a} \log _{e} b / a \times 10^{-7} \text { henry. }
\end{aligned}
$$

$\Delta L_{a}=$ inductance of air space between current sheet and core; $A_{a}$ is the residual area of air space; $d_{e}$ is effective magnetic diameter; and $x$ is the distance of the effective current sheet from the face of the toroid. For $\mu=1$, one may alternatively apply a correction to $L_{s}$ in eq (1), so that the air inductance would effectively be increased to compensate for the larger effective coil cross section. Equation (2) then becomes

$$
L_{S^{\prime}}=2 N^{2}(h+2 x) \log _{e} \frac{b+2 x}{a-2 x} 10^{-7} \text { henry. }
$$

It must be noted at this point that the above corrections account only for effects of finite wire dimensions. The fact that leakage fields do exist beyond the confines of the coil is not considered. A theoretical treatment from the literature of the effects of stray fields will be discussed in a later section, after a consideration of additional experimental results.

\section{Experimental Results}

We define the correction terms $\Delta L_{m}$ and $\Delta L_{S}$, such that

$$
\mu=\frac{L_{m}-\Delta L_{m}}{L_{S}}
$$

or

$$
\mu=\frac{L_{m}}{L_{S}+\Delta L_{S}} .
$$

No geometrical meaning is affixed to either correction term, except that when either is applied, all necessary correction will have been taken into account.

It may be shown from eq (8) and (9) that

$$
\begin{aligned}
& \Delta L_{m}=\Delta \mu L_{S} \\
& \Delta L_{S}=\frac{\Delta \mu L_{S}{ }^{2}}{L_{m}-\Delta \mu L_{S}} \\
& \Delta L_{S}=\frac{\Delta L_{m}}{\mu},
\end{aligned}
$$

which relate the correction terms to measurable quantities.

If now the number of turns on a group of cores of the same size but different permeabilities is varied and the effective permeability, $\mu_{e}$, is plotted as a function of $N^{2}$ (fig. 4), it is observed that within the limits of the test, $\Delta \mu$ is the same for all samples for any particular value of $N$. Thus for a given core size and wire size, $\Delta \mu$ is only a function of the number of turns on the core, i. e.,

$$
\Delta \mu=f(N) .
$$

With this result in mind, a detailed study of the behavior of $\Delta \mu$ is made on a polystyrene core, and it is assumed that the conclusions that will be drawn will be applicable to all other cores of the same physical dimensions wound with the same wire. The reasons for choosing a polystyrene core for this detailed study are

1. $\mu$ is very accurately known $(\mu=1)[5]$.

2. The percentage error $\Delta \mu / \mu$ is largest for the polystyrene core, and may therefore be more precisely determined.

Figure 5 shows the results of another series of measurements on a polystyrene core, similar to those for the magnetic cores, with the effective permeability, $\mu_{e}$, plotted as a function of $N$, with a given core and wire size. It is noted that, indeed, the general shape of the curve is the same as in figures 2 and 4 , and that the value of the asymptotic permeability, $\mu_{a}$, is 6 percent above the true permeability.

If one now assumes a current distribution such that the current is concentrated on the periphery of the wire, and from this deduces the distance $x$ between the current sheet and core face to be half the diameter of the wire, one may apply the correction. terms in eq (7) or (2a), substituting $d / 2$ for $x$. It is observed that both correction terms yield approximately the same result, i. e., the entire curve is shifted down and approaches the value of $\mu_{e}=1.03$ asymptotically. Therefore, this new assumption

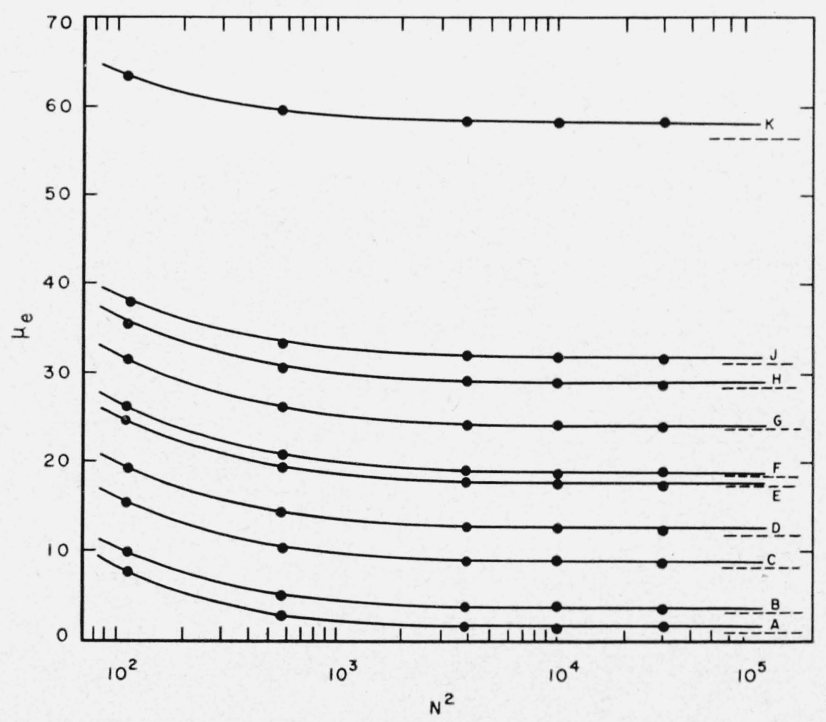

Figure 4. Variation of $\mu_{e}$ as a function of $N^{2}$ with $\mu$ as a parameter for a group of magnetic cores of given dimensions with given wire size.

Dotted lines indicate values of true permeability. Wire: Insulated No. 38. 
decreases the error to 3 percent, but does not account entirely for the error $\Delta \mu$. This indicates that the current distribution in the wire that has now been assumed might be incorrect because of the proximity of neighboring turns of wire, which certainly will influence the distribution. Furthermore, the experimental evidence indicates that the effective current sheet is located at a still greater distance from the face of the core. It might be noted at this point that the problem of winding a wire adjacent to the core face is a difficult one, and it can ba shown that even a small air space will lead to a considerable error $\Delta \mu$.

Figure 6 shows a plot of $\mu_{e}$ as a function of $N$, with wire size as a parameter. It is observed that the basic curve is shifted upward and to the left for increasing wire size. The curves indicate an increase in the value of the asymptotic permeability $\mu_{a}$ with increasing wire size, showing that the current distribution is altered in such a manner as to increase the distance of the effective current sheet for larger wire. At the same time, because of a greater effective "current strip width" per turn, the uniform current sheet is more closely approximated by the heavier wire for small values of $N$. This causes less flux to be lost from the test core by leakage, which in turn results in a smaller $\Delta \mu$. Thus it may be seen that for small $N$, the smaller wire yields a larger $\Delta \mu$, whereas for large $N$, the smaller wire yields the smaller $\Delta \mu$.

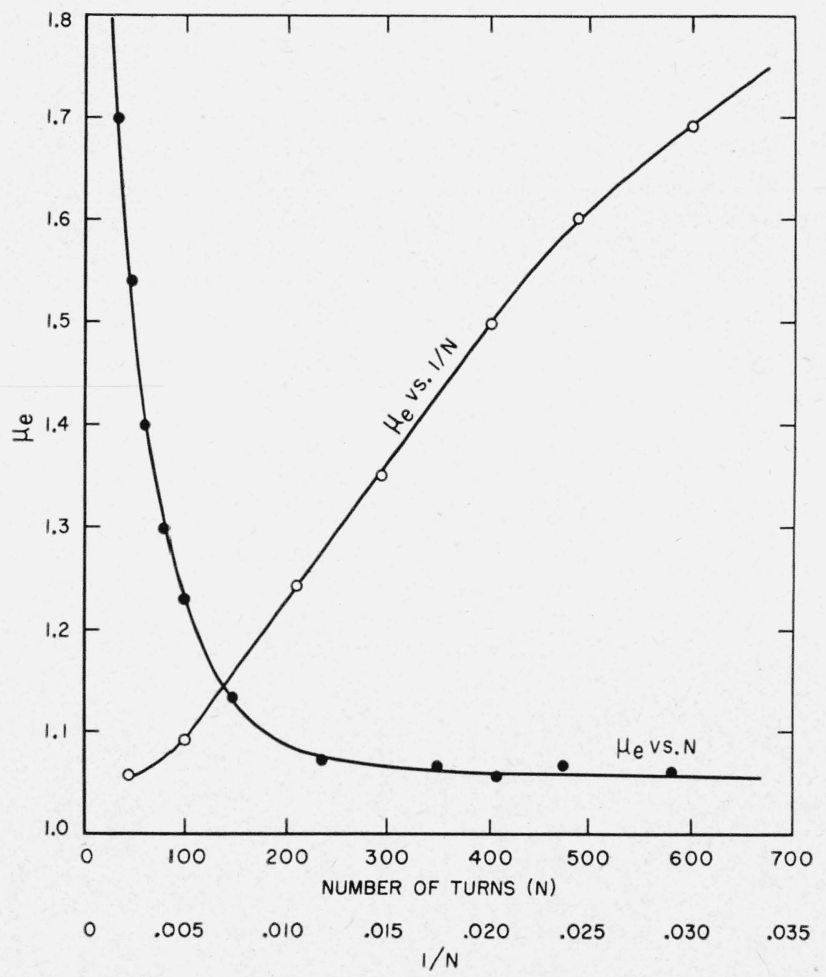

FigURE 5. Variation of $\mu_{e}$ as a function of number of turns $N$ for a polystyrene core.

Wire: Insulated No. 38. Core: Outside diameter, 1.504 in.; inside diameter, 1.004 in.; height, 0.373 in.
If one plots $\mu_{e}$ as a function of $1 / N$ for a polystyrene core (as in fig. 5), it is observed that within limits a linear relationship exists. If, furthermore, the core size is varied (see table 1 ), and $\mu_{e}$ is plotted as a function of $1 / N$, with a given wire size (fig. 7 ), it is observed that the slope is the same for all coils tested within the linear portion. Considering only the linear portion of the curve and choosing the true permeability as a base point, it is concluded that within the limits of the test, $\Delta \mu$ is a linear function of $1 / N$, i. e.,

$$
\Delta \mu=\frac{K_{1}}{N}+C_{1}
$$

Figure 8 shows a plot of $\mu_{e}$ as a function of $1 / N$, with wire size as a parameter; it is observed that the slope $K_{1}$ is dependent on wire size.

$$
K_{1}=f(d) .
$$

\begin{tabular}{|c|c|c|c|}
\hline Coil & $\begin{array}{l}\text { Outside } \\
\text { diameter }\end{array}$ & $\begin{array}{c}\text { Inside } \\
\text { diameter }\end{array}$ & Height \\
\hline $\begin{array}{l}1 \\
2 \\
3 \\
4\end{array}$ & $\begin{array}{l}\text { in. } \\
1.600 \\
1.300 \\
1.000 \\
0.700\end{array}$ & $\begin{array}{c}\text { in. } \\
1.068 \\
0.868 \\
.668 \\
.468\end{array}$ & $\begin{array}{l}\text { in. } \\
0.532 \\
.432 \\
.332 \\
.232\end{array}$ \\
\hline
\end{tabular}

TABLE 1. Dimensions of the cores used in figure 7

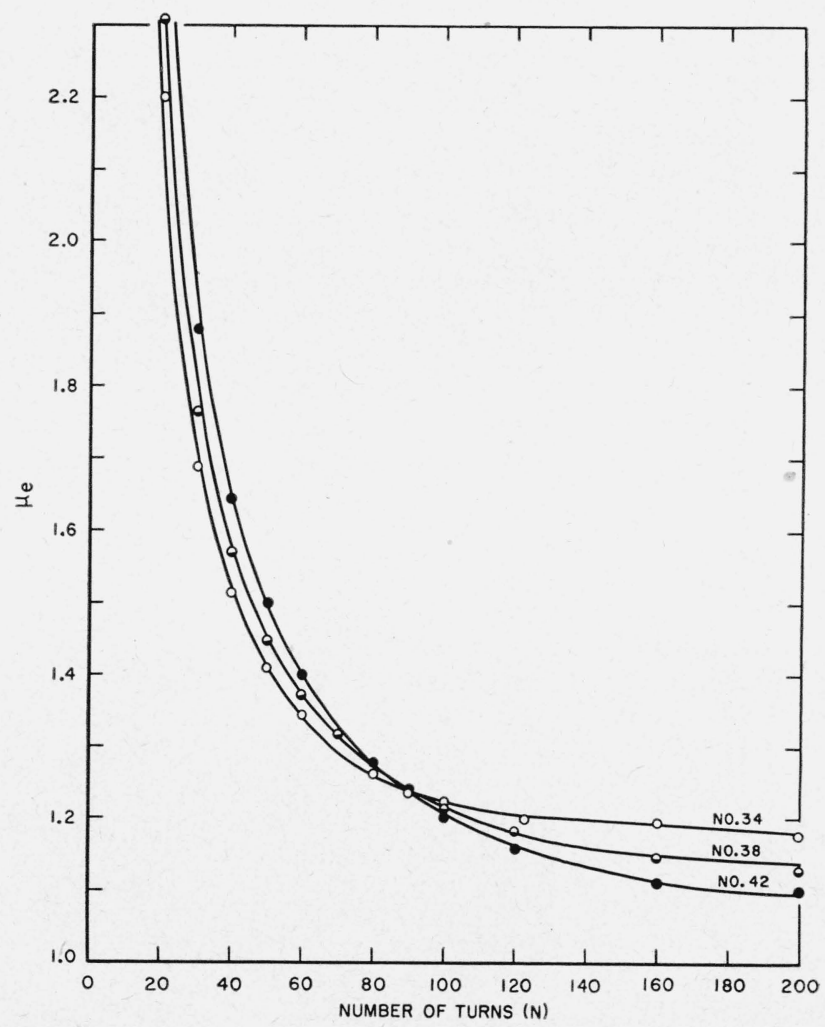

FIGURE 6. Variation of $\mu_{e}$ as a function of number of turns $N$, with wire size as a parameter.

Core: Outside diameter, 1.504 in.; inside diameter, 1.004 in.; height, 0.371 in. 


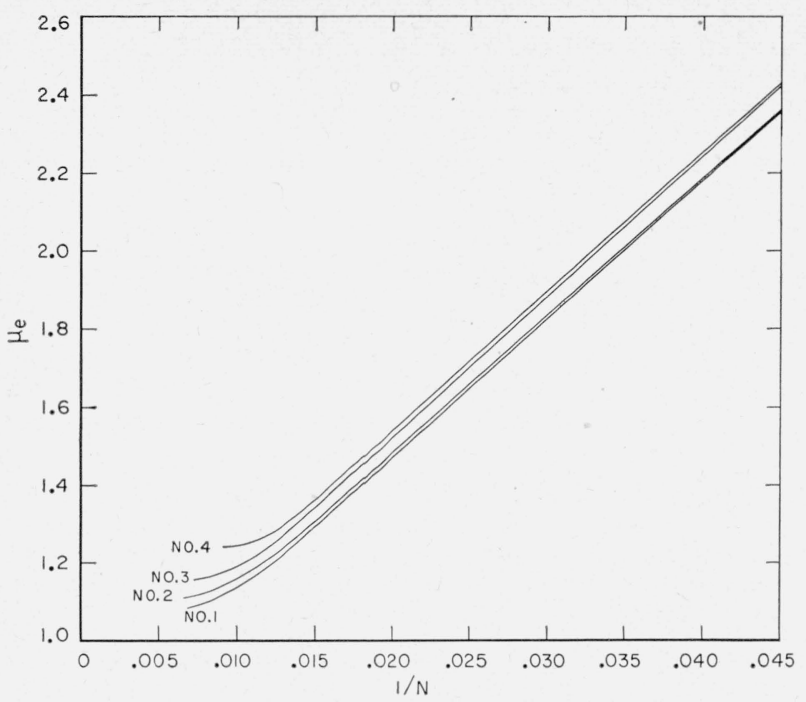

Figure 7. Variation of $\mu_{e}$ as a function of $1 / N$, with core size as a parameter.

Wire: Insulated No. 38. See table 1 for core sizes. Experimental curves; points omitted for reasons of clarity.

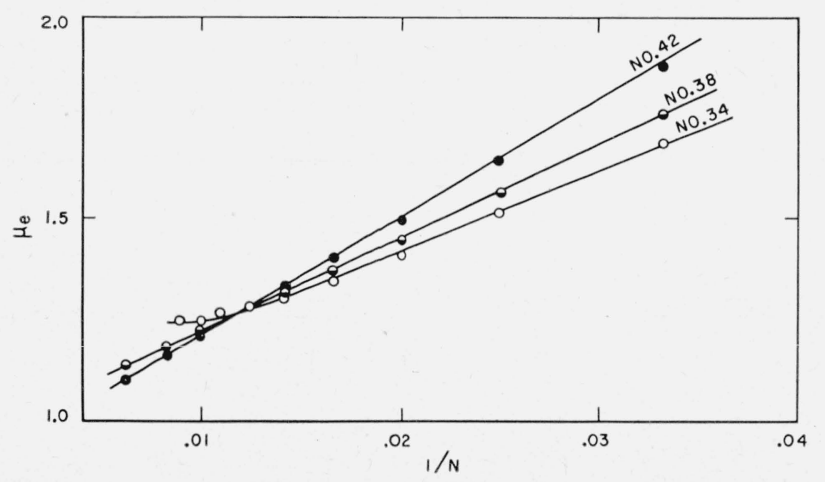

Figure 8. Variation of $\mu_{e}$ as a function of $1 / N$, with wire size as a parameter.

Core: Outside diameter, 1.504 in.; inside diameter, 1.004 in.; height, $0.371 \mathrm{in.}$

The term $C_{1}$ is numerically small by comparison to $K_{1}$ and apparently dependent on both core and wire size.

A mean spacing between wire enters may also be defined by

$$
D=\frac{\pi(b+a)}{2 N}
$$

As $D$ is linearly related to $N$, it may be concluded that within the range stated, the error is a linear function of the mean spacing between wire centers. Thus eq (14) may be written

where

$$
\Delta \mu=K_{2} D+C_{1},
$$

$$
K_{2}=\frac{2 K_{1}}{\pi(b+a)} \text {. }
$$

The data of figure 7 is replotted on figure 9, which shows a plot of $\mu_{e}$ as a function of $D$, with core size

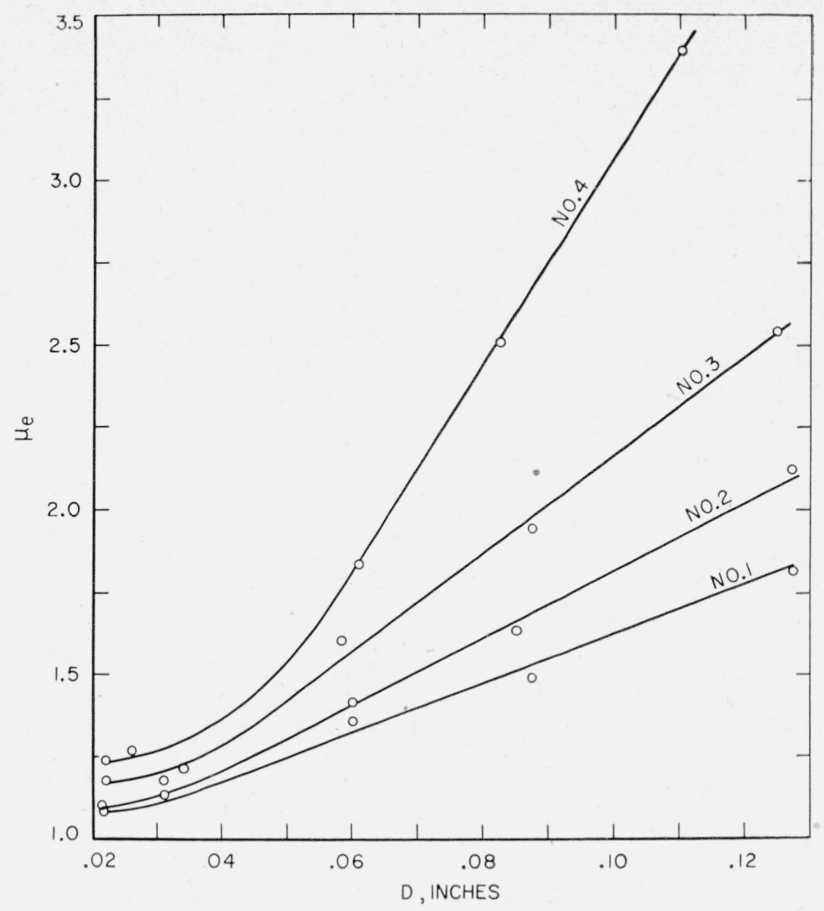

Figure 9. Variation of $\mu_{e}$ as a function of $D$ for various cores (see table 1).

Wire: Insulated No. 38

as the parameter. It is interesting to note that $\Delta \mu$ is largest for the smallest test sample, with a given wire spacing, $D$.

\section{Effects on High-Frequency Magnetic Measurements}

Sufficient experimental evidence has now been presented to allow us to draw some conclusions as to the effect of flux leakage on permeability measurements at high frequencies. The quantity of major interest in an analysis of the accuracy of a magnetic measurement is the fractional permeability error, $E$, given by

$$
E=\frac{\Delta \mu}{\mu}
$$

From eq (14) we have then

$$
E=\frac{K_{1}}{\mu N}+\frac{C_{1}}{\mu}
$$

The values of $K_{1}$ and $C_{1}$ obtained for a number of different core and wire sizes were all such that for the number of turns $N$ usually employed at frequencies in the megacycle region, the error can be approximated by

$$
\frac{\Delta \mu}{\mu} \cong \frac{K_{1}}{\mu N} \cong E .
$$


Under these conditions, one then obtains the condition for constant error

$$
\mu N=\text { constant }
$$

(for a coil with given wire size).

Let it now be assumed that a toroidal core of permeability $\mu_{1}$ has been wound with $N_{1}$ turns, yielding a measured inductance $L_{m_{1}}$. Let it furthermore be assumed that an error $E_{1}$ (this error caused by violation of current-sheet assumption only) results from a calculation of permeability by using eq (1), and that this represents a maximum error that should not be exceeded in the measurement of other cores of permeability $\mu_{n}$ of identical dimensions.

From eq (8) we have

$$
L_{m_{1}}=\mu_{1} L_{S_{1}}+\Delta L_{m_{1}} .
$$

From eq (10) and (23), we have

$$
\left.\begin{array}{rl}
L_{m_{1}} & =\left(\mu_{1}+\Delta \mu_{1}\right) L_{S} \\
& =\left(1+E_{1}\right) \mu_{1} N_{1}^{2} L_{a}
\end{array}\right\}
$$

where $E_{1}=\Delta \mu_{1} / \mu_{1}$ and $L_{S} \equiv N^{2} L_{a}$. Now a core of permeability $\mu_{2}$ is to be measured with an error, $E_{2}$, no greater than $E_{1}$. If we consider the error to be held constant, i. e., $E_{1}=E_{2}$, then from eq (22), we have

$$
N_{2}=\frac{\mu_{1}}{\mu_{2}} N_{1}
$$

We now investigate the value of $L_{m_{2}}$, i. e., the measured inductance of coil 2 , to satisfy the constanterror condition. As in eq (24), we have

$$
\left.\begin{array}{rl}
L_{m_{2}} & =\left(1+E_{2}\right) \mu_{2} N_{2}^{2} L_{a} \\
& =\left(1+E_{1}\right) \mu_{1}^{2} N_{1}^{2} L_{a} \frac{1}{\mu_{2}}
\end{array}\right\}
$$

Dividing eq (26) by eq (24), we have, solving for $L_{m_{2}}$ :

$$
L_{m_{2}}=\frac{\mu_{1}}{\mu_{2}} L_{m_{1}}
$$

This is the value of measured inductance necessary in sample 2 for the error $\Delta \mu_{2} / \mu_{2}$ to equal the error $\Delta \mu_{1} / \mu_{1}$.

If permeability $\mu_{2}$ of core 2 is assumed to be less than that of core 1 , it is noted that for the errors from violation of uniform, thin current-sheet assumptions to be the same, coil 2 must possess an inductance larger than that of coil 1 by the ratio of the two permeabilities.

Up to this point only errors in the determination of high-frequency permeability caused by violating current-sheet assumptions have been considered. Errors, however, are also introduced by the presence of capacitance between the windings of the coil. In particular, if the inductance, $L$, of a coil, and its distributed capacitance, $C$, may be regarded as lumped inductance and capacitance in parallel, the effective inductance of the coil is given by

$$
\begin{gathered}
L_{e}=\frac{L}{1-\omega^{2} L C}=\frac{1}{1-\left(f / f_{0}\right)^{2}}, \\
f_{0}=\frac{1}{2_{\pi} \sqrt{L C}},
\end{gathered}
$$

where $L_{e}$ is effective inductance; $L$ is actual inductance; $f_{0}$ is the self-resonant frequency of the coil. In order for the measurement to remain reasonably free from errors caused by distributed capacitance, as shown in eq (29), the measurement frequency, $f$, must be much lower than the natural resonant frequency, $f_{0}$.

Considering the case discussed above, wherein the error $\Delta \mu / \mu$ for cores of permeability $\mu_{1}$ and $\mu_{2}$ is to be constant, $\mu_{2}$ being less than $\mu_{1}$, it is noted that coil 2 must have a higher inductance than coil 1 . However, in order to have this larger inductance, more turns had to be placed on core 2 than on core 1 , resulting in an increase of capacitance for coil 2 . Consequently, coil 2 will have a lower natural resonance frequency $f_{0}$ than coil 1 because of both higher inductance and capacitance. It thus appears that a smaller measurement error will result for a higher permeability at a given frequency.

It has been shown by numerous authors [2] that the effects of distributed capacitance may be corrected for by measuring the self-inductance at several frequencies. It must be pointed out, however, that this procedure assumes a constant self-inductance and thus a constant permeability with frequency. If this, however, be known a priori, a meausrement at a frequency where errors from residual capacitance may be neglected would suffice.

\section{Experimental Evaluation of Theoretical Corrections}

As noted previously, attempts have been made to correct the approximate eq (1) for the assumptions made.

In the formulation already described, it is inferred that there are no leakage fields existing beyond the confines of the coil. If this were so, then the correction $\Delta L_{a}$ in eq (6) would equal the correction $\Delta L_{m}$. From eq (7), we see that

$$
\Delta L_{a}=C N^{2} .
$$

It has been shown in eq (14) that

$$
\Delta \mu=\frac{K_{1}}{N}+C_{1}
$$

But we also have from eq (10) and from the identity $L_{S}=N^{2} L_{a}$,

$$
\Delta L_{m}=\Delta \mu N^{2} L_{a}
$$


From this equation and eq (14), we have

$$
\Delta L_{m}=K_{3} N+K_{4} N^{2} .
$$

For all but large values of $N, \Delta L_{m}$ is dominated by the term $K_{3} N$, and only for very large $N$ is $\Delta L_{m}$ more nearly a function of $N^{2}$. Therefore, it may be concluded that eq (30) will apply only at large $N$, where the assumption that a uniform current sheet exists is more nearly valid. However, for highfrequency measurements it is important, as stated, to keep the distributed capacitance down to a minumum value, which is seldom feasible if a constant, uniform current sheet is simultaneously required.

Another correction based on a theoretical approach takes into account the possibility of leakage fields existing, when the core is wound with round wire of finite dimensions. Under such conditions the selfinductance is affected by those lines of flux within the wire and by those linked with each separate turn of wire in addition to those lines of flux within the test core. The correction worked out by Rosa alters the calculated value of inductance $L_{s}$. The correction term depends on the pitch and size of wire and may be positive or negative. It is determined by considering the difference in self-inductance and mutual inductance between turns of a coil of round wire of finite dimensions from those of a coil of thin tape.

Rosa's equation for the self-inductance of an air toroid is [4]

$$
\begin{gathered}
L=L_{S}-2 N i(A+B) \\
A=\log _{e}\left(1.745 \frac{d}{D}\right),
\end{gathered}
$$

where $l$ is the length of one turn of wire equals $2(h+b-a) ; d$ is the diameter of the wire used; $D$ is the mean wire spacing (see eq 16); $B$ is the correction from Grover's tables [6] for mutual inductance between turns. We define Rosa's correction term

$$
\Delta L_{R}=-2 N l(A+B) .
$$

It is noted at this point that if $\Delta L_{R}$ included all of the necessary corrections, it should equal the correction term $\Delta \dot{L}_{S}$ for an air toroid, (see eq 9 ). We thus consider a polystyrene core and from the observed data, plot $\Delta L_{S}$ as given by eq (11).

$$
\Delta L_{S}=\frac{\Delta \mu L_{S}^{2}}{L_{m}-\Delta \mu L_{S}} .
$$

If one uses $L_{S}$ as defined by eq (2) to determine $\Delta L_{S}$, a plot of $\Delta L_{S}$ (see top curve of fig. 10) as a function of $N$ does not compare favorably with a plot of the Rosa correction term $\Delta L_{R}$. Furthermore, if we substitute a corrected value of $L_{S}$, assuming the current sheet to be a distance $d / 2$ from the sample surface, it is noted that the agreement is but slightly improved. Recalling our earlier prediction as to separation of permeability errors into two parts, and

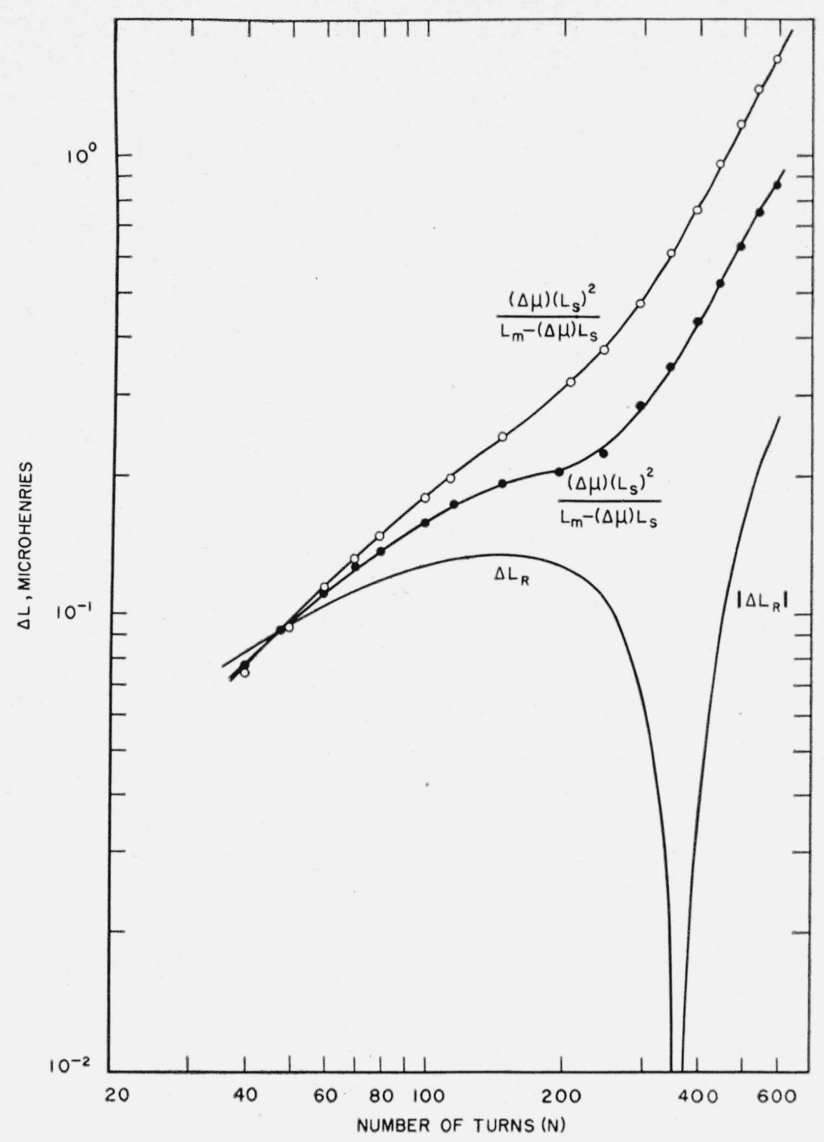

Figure 10. Comparison of Rosa correction term with experimental data.

$\Delta L_{R}$ is the absolute value of Rosa correction term.

on the basis that $\Delta L_{R}$ takes care of nonuniformity of current sheets only, we calculate $\Delta L_{S}$ by using $\Delta \mu_{2}$ as shown:

$$
\Delta L_{S}=\frac{\Delta \mu_{2} L_{S}^{2}}{L_{m}-\Delta \mu_{2} L_{S}} .
$$

We now plot this equation as a function of $N$ (see fig. 11) and notice that a favorable comparison with Rosa's correction term $\Delta L_{R}$ is obtained.

As a result of eq (10) and (12) and the experimental fact that $\Delta \mu$ is not a function of $\mu$, we find that

$$
\Delta L_{S}=\frac{\Delta \mu L_{S}}{\mu} .
$$

Thus the values of $\Delta L_{S}$ for two cores of permeabilities, $\mu_{1}$ and $\mu_{2}$, are related by the equation

$$
\Delta L_{S_{2}}=\frac{\mu_{1}}{\mu_{2}} \Delta L_{S_{1}}
$$

While Rosa's correction term is strictly valid only for an air toroid, it was pointed out earlier that in the calculation for an air toroid it takes the place of our correction term $\Delta L_{S}$. In comparing its possible 


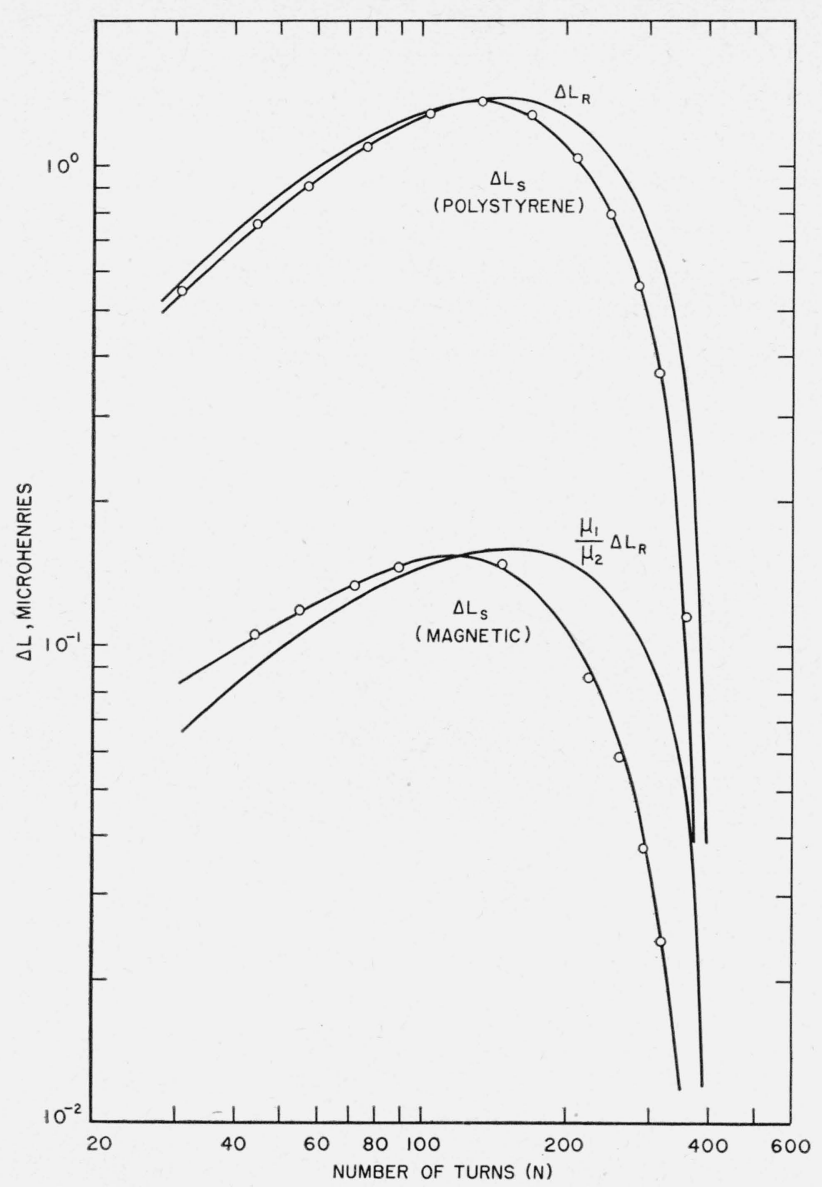

Figure 11. Comparison of Rosa correction term with experimental data.

validity for a coil with arbitrary permeability $\mu$, we thus let it take the place of $\Delta L_{S_{1}}$ in eq (37), with $\mu_{1}$ now equal to unity. The two curves, that is, $\Delta L_{S}$ for a magnetic core and $\Delta L_{R}$ divided by that core's permeability, are shown plotted versus $N$ as the bottom curves on figure 11. The fairly good agreement obtained indicates a possibility of estimating a value of the permeability for a magnetic core by using Rosa's correction term in the manner indicated.

We have now shown the effects of two possible calculations of correction terms. Referring once more to eq (3),

$$
\Delta \mu=\Delta \mu_{1}+\Delta \mu_{2}
$$

it may be stated that the error $\Delta \mu_{1}$ is indeed associated with violation of the assumption that the current sheet is adjacent to the toroidal sample. As shown, this may be accounted for by eq (7) or (2a), provided one knows the distance $x$ from the face of the test sample to the location of the effective current sheet. The value of $\Delta \mu_{2}$ may be associated with the error caused by the presence of leakage fields beyond the confines of the coil. This error may be estimated, at least for an air coil and within limits for any coil, by applying the Rosa correction term.

\section{Permeability Measurement by Air-Coil Substitution}

Another method mentioned earlier for measuring high-frequency permeability utilizes the measured inductance of a coil wound on a nonmagnetic core. This coil is to have the same physical dimensions as the magnetic core whose permeability is to be determined and wound with the same number of turns and wire. For the permeability, one then takes

$$
\mu_{e}=\frac{\left(L_{m}\right)_{m}}{\left(L_{m}\right)_{a}} .
$$

However, by referring to eq (8) and (12), we have

$$
\begin{aligned}
& \left(L_{m}\right)_{m}=\mu\left(L_{S}+\Delta L_{S_{m}}\right) \\
& \left(L_{m}\right)_{a}=(1)\left(L_{S}+\Delta L_{S_{a}}\right),
\end{aligned}
$$

and eq (38) becomes

$$
\mu_{e}=\frac{\mu\left(L_{S}+\Delta L_{S_{m}}\right)}{L_{S}+\Delta L_{S_{a}}} .
$$

Only when $\Delta L_{S_{a}}=\Delta L_{S_{m}}$ will this ratio reduce to $\mu$. On the basis of the experimental evidence and eq (10) and (12), one obtains

$$
\begin{aligned}
\Delta L_{S_{m}} & =\frac{\Delta \mu}{\mu} L_{S} \\
\Delta L_{S_{a}} & =\Delta \mu L_{S}
\end{aligned}
$$

Equation (41) becomes, upon substituting the results of eq (42) and (43),

$$
\mu_{e}=\frac{\mu(1+\Delta \mu / \mu)}{1+\Delta \mu}
$$

As $\Delta \mu \neq \Delta \mu / \mu$, unless $\mu=1$, $\mu_{\text {eff }}$ will always lead to values on the low side, the absolute error becoming larger for higher permeability. A practical difficulty that may be encountered is that it is extremely difficult to wind coils exactly alike, and thus further errors might be introduced.

\section{Experimental Details}

The magnetic cores used for this investigation were of powdered iron. Field strengths above 1 oersted are usually required to effectively change the permeability of such cores; the field strengths employed were below 0.01 oersted in all cases. Care was taken to assure that changes in the field intensity by factors as large as 2 to 1 would not change the coil inductance by even as much as 1 part in 10,000 . 
The frequency used was $50 \mathrm{kc}$. The coil with the highest reactance used in this investigation was measured in the range from 1 to $50 \mathrm{kc}$. The effective inductance showed a change of less than 1 part in 10,000 , which could be ascribed to capacitance effects.

The bridges used for the measurements were two high-quality Maxwell-type inductance bridges designed at Bell Telephone Laboratories. Connections between the unknown terminals of the bridges and the coils were made in such a manner that proximity effects from neighboring objects were held to a negligible minimum and that the lead inductance could be accounted for.

The true initial permeabilities of the magnetic cores were determined by a specially calibrated radio-frequency permeameter used in conjunction with calibrated capacitors.

In order to apply the windings as uniform and tight as possible without any crossovers whatever and no visible injury to the insulation, a toroidal coil winder capable of applying this type of singlelayer winding was employed. Figure 1 shows an example of such windings.

Washington, January 7, 1954.

\section{Summary}

The errors encountered in high-frequency magnetic measurements have been discussed mostly from the viewpoint of violation of uniform, thin current-sheet assumptions. The absolute error in permeability has been shown to be independent of the permeability of the material tested, and its dependence on the spacing between turns has been formulated. The adequacy of certain correction terms suggested by other authors have been investigated. It has been shown that an approximate correction may be made utilizing a combination of several such terms, provided the extension of the leakage flux into space may be estimated.

\section{References}

[1] V. E. Legg, Magnetic measurements at the low flux densities using the A. C. bridge, Bell System Tech. J. 16, 39 (1936).

[2] L. Hartshorn, Radio frequency measurements by bridge and resonance methods, p. 231 (Chapman \& Hall, Ltd., London, 1941).

[3] P. H. Haas, A radio-frequency permeameter, J. Research NBS 51, 221 (1953) RP2454.

[4] Formulas and tables for the calculation of mutual and self-inductance (Revised), NBS Sci. Paper 169, p. 125 (1912).

[5] International critical tables, vol. VI (1929).

[6] F. W. Grover, Inductance calculations, p. 148 (Van Nostrand \& Co.). 